\title{
Unifying Cloud Management: Towards Overall Governance of Business Level Objectives
}

\author{
Mina Sedaghat, Francisco Hernández, Erik Elmroth \\ Dept. of Computing Science, Umeå University, Sweden \\ \{mina, hernandf, elmroth\}@cs.umu.se
}

\begin{abstract}
We address the challenge of providing unified cloud resource management towards an overall business level objective, given the multitude of managerial tasks to be performed and the complexity of any architecture to support them. Resource level management tasks include elasticity control, virtual machine and data placement, autonomous fault management, etc, which are intrinsically difficult problems since services normally have unknown lifetime and capacity demands that varies largely over time. To unify the management of these problems, (for optimization with respect to some higher level business level objective, like optimizing revenue while breaking no more than a certain percentage of service level agreements) becomes even more challenging as the resource level managerial challenges are far from independent. After providing the general problem formulation, we review recent approaches taken by the research community, including mainly general autonomic computing technology for large-scale environments and resource level management tools equipped with some business oriented or otherwise qualitative features. We propose and illustrate a policy-driven approach where a high-level management system monitors overall system and services behavior and adjusts lower level policies (e.g., thresholds for admission control, elasticity control, server consolidation level, etc) for optimization towards the measurable business level objectives.
\end{abstract}

Keywords: Cloud governance; Autonomic Computing; Policy-driven Management.

\section{INTRODUCTION}

Cloud management for optimizing a cloud provider's overall objectives is becoming increasingly more difficult following the rapidly increasing scale of resources and services to be managed and the intrinsic challenges of fundamental cloud management. Already individual fundamental cloud management issues such as admission and elasticity control, virtual machine (VM) and data placement, autonomous fault management, etc. are difficult since services normally have unknown lifetime and capacity demands that vary largely over time.

The overarching management goal of cloud infrastructure providers (IPs) is often referred to as the managerial Business Level Objective (BLO). For a commercial IP this BLO may, for example, be to manage resources so as to optimize revenue $^{1}$ while keeping customers sufficiently happy ${ }^{2}$. For a noncommercial IP, the BLO may, e.g., be expressed in terms of

\footnotetext{
${ }^{1}$ Revenue calculation can be arbitrary complicated but should at least take into account parameters such as income, operation and infrastructure costs and costs associated with violated service level agreements (SLAs).

${ }^{2}$ For resource management, a criterion such as keeping customers sufficiently happy may, e.g., be translated into constraints on maximum fractions of SLAs to violate.
}

maximizing resource utilization while maintaining a specified level of fairness between users [1].

The overall management problem becomes the complex challenge of managing the individual tasks of admission and elasticity control, VM and data placement, fault management, etc, and to optimize the overall system behavior with respect to the BLO. An attractive way of dealing with the complexity is to separate functionalities into individual and to some degree autonomous components with clear separation of concerns, e.g., including separate components for admission control, elasticity control, VM placement, data placement, fault management, etc. However, these problems are not totally independent. For example, increasing the server consolidation level (number of VMs per physical host) or making the elasticity control more restrictive would allow for admission control to be less restrictive in accepting services. Hence, there is a need for some degree of coordination between such actions. In this work we call this coordination governance. This problem is further examined in Section II.

Recent studies on this type of management issues for clouds are mainly focusing on either the general autonomic computing aspects supporting management of truly large scale environments or on how to enhance cloud resource level functionalities by adding business or qualitative features. Section III reviews several existing solutions on management in clouds with respect to business level concerns. The review is followed by a description of current challenges in designing a business oriented governance model, presented in Section IV. Section V proposes a governance model that aim to direct and control a set of lower level management tools so that their concerted actions strive towards the overall BLO. Finally, Section VI concludes the paper and outlines possible future research directions.

\section{CLOUD MANAGEMENT}

Current efforts in cloud resource and service management mainly focus on the most technical and fundamental aspects of resource provisioning [2]. Managerial expectations and business objectives, although important, are seldom considered. One such expectation is efficiency in resource utilization with respect to SLA constraints and QoS requirements. Under normal circumstances services are not using their maximum required capacity all at once. Hence, in order to efficiently make use of available resources, infrastructure providers aim to sell more capacity (with respect to services expected peak 
loads) than what is physically available, i.e., controlled overprovisioning $^{3}$. To complicate matters, there are other challenges that need to be considered like the necessity to support elastic behaviors of services without breaking SLAs or SLA management mechanisms that prioritize services when not all SLAs can be met. Furthermore, mechanisms to avoid crashes and failures i.e., manage breakdowns and repair actions, are also needed. Key to handle these types of cloud management challenges is the ability to provide efficient and coordinated solutions to the following problems:

- Admission Control (AC): IPs should be able to decide whether a service should be accepted or rejected taking economic and other considerations into account.

- Elasticity: IPs should be able to predict and manage the elastic behavior of services. Rapid changes in service demands should be handled by appropriate capacity adjustments, so that services can scale up or scale down easily.

- Fault tolerance: IPs should be able to manage crashes and infrastructure failures. Unexpected resource failures should be managed through repair actions while prediction of possible upcoming crashes, in order to facilitate preventive actions, should also be considered.

- Data placement: IPs should be able to place data at optimal resources taken into account, e.g., a range of performance aspects and resource availability.

- VM placement: IPs should be able to place VMs on physical machines based on their SLAs and on an estimation of their future behaviors. Efficiency in resource utilization should also be taken into account.

- SLA management: IPs should be able to enforce SLAs and manage violations based on priorities.

All of these challenges indicate specific problems that can be formulated and solved more or less independently. However, although each problem can be solved autonomously to satisfy its specific goals it should be acknowledged that the problems are intertwined. Table I, summarizes the aforementioned problems and introduces sample software components (from here on called low level managers) to address them. The table also shows the information required to tackle the problem as well as the outcomes expected from each low level manager. The outcomes can be a decision making or a specific action.

Ideally, all these problems should be solved in concert, striving towards a high level managerial objective, i.e. the resource management BLO. BLOs delineate a desired goal like maximizing profit without breaking more than a certain fraction of SLAs or (for a non-profit organization) to maximize resource utilization while maintaining fairness among users. Part of the challenge is that an optimal solution to one of the specific management problems may be in conflict with the concerted actions needed to optimize the BLO. For example, an AC can accept services as long as there are available

\footnotetext{
${ }^{3}$ The term typically used is over provisioning. However, due to the possible negative connotation of the term, we prefer to explicitly state that the over provisioning is controlled, i.e., it is in line with the business goals of cloud providers.
}

TABLE I: Important cloud management challenges: Logical view. Notice that a real implementation may address several of these challenges in a single software component

\begin{tabular}{|c|c|c|}
\hline $\begin{array}{l}\text { Low level } \\
\text { manager }\end{array}$ & Required information & $\begin{array}{l}\text { Activities and Decision mak- } \\
\text { ing }\end{array}$ \\
\hline $\begin{array}{l}\text { Admission } \\
\text { Controller }\end{array}$ & $\begin{array}{l}\text { 1.The available Capacity } \\
\text { 2.Expected load from all services } \\
\text { 2.SLA requirements per service } \\
\text { 3. Historic workload } \\
\text { 4.Price tariffs }\end{array}$ & $\begin{array}{l}\text { 1.Accept or Reject } \\
\text { 2.Select a service } \\
\text { from a queue }\end{array}$ \\
\hline $\begin{array}{l}\text { VM Placement } \\
\text { Engine }\end{array}$ & $\begin{array}{l}\text { 1.The total amount of resources } \\
\text { 2.The allocated resources } \\
\text { 3.SLA including placement } \\
\text { constraints } \\
\text { 4.Data locality }\end{array}$ & $\begin{array}{l}\text { 1.Service placement } \\
\text { 2.Service termination } \\
\text { or cancellation } \\
\text { 3.Service migration }\end{array}$ \\
\hline $\begin{array}{l}\text { Data } \\
\text { Placement } \\
\text { Engine }\end{array}$ & $\begin{array}{l}\text { 1.Current and predicted storage } \\
\text { 2.End user locality including } \\
\text { trends,network performance } \\
\text { and characteristics }\end{array}$ & $\begin{array}{l}\text { 1.Data objects placement } \\
\text { 2.Data objects migration }\end{array}$ \\
\hline $\begin{array}{l}\text { Elasticity En- } \\
\text { gine }\end{array}$ & $\begin{array}{l}\text { 1.Current load } \\
\text { 2.Predicted future load } \\
\text { 3.Historic load and trends } \\
\text { 4.SLAs }\end{array}$ & $\begin{array}{l}\text { 1.Allocate additional resource } \\
\text { 2.Release allocation }\end{array}$ \\
\hline $\begin{array}{l}\text { Fault } \\
\text { Tolerance } \\
\text { Controller }\end{array}$ & $\begin{array}{l}\text { 1.Resource status } \\
\text { (Up, Failed, Probate) } \\
\text { 2.List of crashed resources } \\
\text { 3.List of crashed services } \\
\text { 4.Possible repair action }\end{array}$ & $\begin{array}{l}\text { 1.Restart the service } \\
\text { 2. Terminate the service } \\
\text { 3.Postpone the restart } \\
\text { 4.Preventive service migration }\end{array}$ \\
\hline $\begin{array}{l}\text { SLA Manage- } \\
\text { ment Engine }\end{array}$ & $\begin{array}{l}\text { 1.SLA } \\
\text { 2.Service state } \\
\text { (e.g., the expected service } \\
\text { completion time,service deadline) } \\
\text { 3.Penalty per service }\end{array}$ & $\begin{array}{l}\text { 1.SLA prioritizing } \\
\text { 2.SLA violation cost estimation }\end{array}$ \\
\hline
\end{tabular}

resources, selecting between incoming services based on a first come first serve policy. An AC can also overbook resources without considering elastic behaviors of services, that are already running. In this case, even though the AC completely fulfills its own responsibilities, the outcome of the applied policy may not result in the revenue expected by the business manager. This may cause costly SLA violation penalties and reputation damages, both aspects affecting the BLOs. This is due to the AC's lack of interaction with other components like the SLA manager, or lack of information about economic factors like pricing.

Meanwhile, the elasticity controller can detect increases in service demand and it can decide to allocate extra resources to services. However, if this is done in isolation, there may not be enough resources available, if e.g., the placement engine also deploys new services. Deciding whether to increase or decrease the allocation of additional resources or to reject the deployment of the new service involves an evaluation of the impact of either action on the BLOs. As this overarching evaluation is in conflict with the goals of each manager, it requires a higher coordination. To summarize, the variety in business level objectives and strategies, and the need for scalable solutions to support diverse scenarios in a cloud environment illustrate the necessity of an autonomic higher level governance mechanism to handle these complexities. 
The result of having a high level mechanism is an increase in the abstraction level of cloud management in which all high level decision making and BLO management is performed by a higher level governance manager [3], [4], [5]. This manager may adjust the behavior of low level managers with respect to the information collected from all resource level functionalities with the aim of satisfying the high level BLOs.

Advances in the operation of the low level managers are also needed. In the next section we present existent solutions on management approaches for enhancing the normal operation of low level managers with business level concerns, as well as other, more comprehensive, approaches for cloud management that relate to our work.

\section{CLOUD MANAGEMENT SURVEY}

Different aspects of cloud management are partially studied in a number of research projects. In these projects, the problem is mainly addressed by adopting business concerns by a specific low level manager like VM placement engine or admission controller; formulating and solving optimization problems; or autonomic management.

\section{A. Adoption of business concerns by low level managers}

The low level managers presented below may all be considered traditional low level managers enhanced with features to optimize management with respect to some higher level management objective. These enhanced managers are designed to operate independently of other managers based on their own (self-centered) objectives, but the overall coordination in the system remains an unsolved issue.

Puschel et al. [6] focus on developing an admission control mechanism aimed to increase the revenue for an IP. The decision to accept or reject a service is made based on predefined policies for dynamic pricing and client classification. They develop a policy-based decision model by defining policies as heuristics when the environment is non-deterministic and there is not enough information about upcoming services. The policies are defined in terms of SLA's committed, previous workloads, utilization trends, and BLO's.

Perez et al. [7], [8] address cloud scheduling and elasticity problems with respect to end users' satisfaction. Responsiveness and fair share are the adjustable high level objectives supported by the model. They propose a reinforcement learning approach for a resource allocation mechanism, because of its flexibility to adapt its decisions to the elastic behavior, QoS requirements and also the minimal knowledge requirement about the environment. The adaptability and optimal policy selection in this model has been achieved using an on-policy learning algorithm, called SARSA.

Moon et al., in a paper on cloud resource scheduling [9], argue that the success of clouds depends on QoS factors and cost management. Therefore, they propose an SLA aware resource scheduler that minimizes SLA penalty costs and optimizes profit based on a cost heuristic. The scheduler evaluates a number of jobs in the queue individually and picks the jobs in order of priority. The cost based scheduler uses a probability density function for computing the expected cost.

Optimizing fault tolerance and repair mechanisms for cost optimization and improvement of QoS and availability for an IP is addressed by Goldszmidt et al. [10]. They propose a framework for evaluation and optimization of policies governing automated repair services, adopting a two phase approach. The first phase estimates the effectiveness of each repair action by assessing historic data. The second phase uses the information processed in the first phase to refine the policy using machine learning techniques.

There are also a number of projects addressing BLOs in general, not focusing on a specific functionality but in supporting managerial objectives with respect to lower level constraints. SORMA [11] strives to fulfill the provider's ambitions on maximizing revenue and other business type objectives. In SORMA, the business management role is assigned to the EERM framework (Economically Enhanced Resource Manager) [12]. The EERM is a resource manager enhanced with business related features that addresses resource scheduling with respect to SLA management and admission control. The EERM makes use of a rule based policy manager to support adaptable policies that are formulated in Semantic Web Rule Language (SWRL). The overall aim of the EERM is to isolate SORMA's economic layers from the technical ones and orchestrate both economic and technical goals in order to achieve maximum economic profit (i.e. revenue) and resource utilization [6].

The Grid-Econ project [13], [14] takes a broader view on business level concerns. Grid-Econ is essentially a resource broker built on a matching algorithm that considers quantity of resource units, the period of time over which the resource is required or available, the minimum selling price or the maximum buying price, and the expiration date of the request to buy or sell a resource [15]. Different types of risks and trust issues in grid markets to support non-commercial grid stakeholders such as insurance against resource failures, resource quality assurance, stable price offering and capacity planning are also addressed. However, all these qualitative objectives are defined as fixed goals in an auctioning strategy and are not flexible or easily extendable.

\section{B. Optimization problems}

Of interest are also optimization methods or algorithms that satisfy a business objective. Most of these algorithms work around optimization techniques and utility functions. Salehi and Buyya, [16] use a time and cost optimization for resource scheduling. They introduce two market oriented policies that consider user constraints such as time and budget. The produced schedules support the elastic behavior of services, load changes in services, and aim at satisfying deadlines by extending the computational capacity of local resources via hiring resources from cloud providers. Similarly, Silva et al. [17] propose a heuristic algorithm for dynamic resource allocation, with the same constraints on time and cost. The 
algorithm determines the optimal number of hosts for short lived tasks.

Paton et al. [18] demonstrate the use of utility functions in utility driven workload executions in clouds. Utility functions quantify and rank the relevance and desirability of each system state, i.e., the functions offer a common and consistent scale to compare the states and objectives. The states may represent response time, number of QoS goals met or income. The utility metrics discussed in this work are profit and response time. They also introduce an autonomic workload mapper responsible to assign tasks to available execution sites and to dynamically monitor and modify assignments during workload execution. The assignments are based on feedback on the overall progress of submitted requests.

\section{Autonomic management}

Autonomic management offers a more comprehensive approach to cloud management. The goal is to offer functionalities for self-configuration, self-management and selfhealing. The idea is that by adding autonomic features to a system, the system would be able to adapt itself to real time configuration and changes. The general autonomic computing aspects support management of truly large-scale environments.

Unity [19] is an agent based autonomic data center with improved behaviors for a self-manageable computing system. It is structured as a set of autonomic components that can manage themselves and also interact autonomously with humans or other autonomic components. The addressed scenario is an elastic resource allocation between different clusters in a grid. The adaptation of the system to the high level policies is derived by a decomposition of the main policy represented as a utility function. An extension to the Unity Project, IBM Tivoli Intelligent Orchestrator (TIO) [20], [21] is an automated provisioning manager for Internet data centers. TIO is capable to automatically deploy and dynamically optimize resources in response to business objectives in heterogeneous environments [20], and it performs on-demand deployment by proactively sensing and responding to peaks in demand and allocating IT resources to the most important processes based on business policies [20]. Elasticity is supported with respect to load, bandwidth, CPU utilization, etc. through the use of a policy manager.

CERAS laboratory [3], [4], [22], [23] works on different aspects of business driven cloud architecture. They automate several activities like monitoring, analysis and prediction, planning and execution through a feedback loop controller that optimizes specific goals and constraints associated with the type of provider or service models, i.e., IaaS, PaaS, SaaS. The main objective that is studied is cost optimization which is a common interest between the three mentioned service models. Notably, [23] goes deeper by considering response time and mean throughput constraints, while taking into account resource contention.

FoSII (Foundation of Self-Governing ICT Infrastructure) [5] tries to develop a self manageable cloud environment that is autonomously complying with user requirements in terms of SLAs and also it achieves a level of flexibility. The main goal is avoidance of SLA violations and, to this end, advanced monitoring and knowledge management [24], [25] are required. FoSII uses a framework that senses infrastructure resource metrics and predicts the risk of SLA violations based on pre-defined thresholds and actual usage. In order to do this, the SLA parameters are refined and mapped into resource level metrics. These refinements are done based on pre-defined mapping rules defined through the use of Domain Specific Languages (DSLs).

\section{CHALLENGES OF CLOUD GOVERNANCE}

As described above, we view the cloud management problem as a set of management tasks that are addressed by independent managers. In this work, we propose to use cloud governance for coordinating the independent managers for overall optimization of the BLO. Developing the governance solution brings about several challenges classified in 4 main categories: BLO formulation; BLO interpretation to resource level objectives; policy enforcement on resource level engines and monitoring and feedback control.

\section{A. BLO formulation}

Current solutions in BLO formulation mostly express BLOs as fixed goals and formulate the management problem as a set of utility functions with multiple constraints. Cost, profit, and time optimizations are the most popular objectives employed [6], [9], [16], [18] but qualitative aspects such as fairness, responsiveness and utilization have also been considered [7], [8], [10]. However, fixed goals are unable to express the wide range of desired system behaviors that vary from one business to another [19]. For example, commercial providers focus on maximizing profit and reducing costs whereas fairness and utilization may be the most important features for an academic resource provider. Thus, a cloud management system should be adjustable and adaptable to a wide range of economic strategies.

\section{B. BLO interpretation to resource level objectives}

Policies map BLOs to system actions [26]. They translate BLOs into desired resource level behaviors. Several mechanisms have been employed to perform such translations. Emeakaroha et al. [24] employ a mapping table to translate SLAs into quantifiable resource level metrics, e.g. availability is mapped to a function of the time it takes for a failure to be repaired and the time system is available. Unity [19], Paton et al. [18] and Li et al. [27] make use of utility-function based policies that allow the human administrator to guide the operation of the system. The adaptation of the system to the high level policies is made through a decomposition of the utility function into sub-problems, and optimization of these sub-problems directs the resource level components toward the main objectives.In EERM [12], policies are formulated in the semantic web rule language (SWRL). All features of the EERM require the components to be able to communicate 
with the policy manager and base their decisions on the corresponding policies [12].

However, to define a relation between high level objectives and constraints to the system level metrics is not always possible, e.g., the number of policies may be unmanageable if the high level objectives vary frequently.

\section{Policy enforcement on resource level engines}

The overall goal of a governance engine is to direct and adjust the behavior of resource level managers. The capability of the system to adopt the policies is crucial for the operation of the governance manager since resource level managers are the actual enactors of the policies. To this end the governance manager must be able to exert action over the resource level managers. Furthermore, the governance manager adjusts the operation of the system by selecting appropriate policies to enforce, from the policy set of each of the resource level actuators, according to the operation of the system at any given moment and the expected BLOs.

\section{Monitoring and feedback control}

The impact of policy enforcement on the low level engines and on performance of the system as a whole should be monitored and analyzed. The monitoring information enable the system to learn from past behavior, predict future actions, and make appropriate trade offs when selecting policy actions [28]. Changes in the environment, policy adherence and violations, and changes in the system status after enforcing a policy may repeatedly trigger new policy settings. The monitoring system gathers information about events and collects performance metrics required for the governance process. The governance manager can analyze the monitored information,e.g., to find correlations between management events, system status and the expected performance. These correlations identify the actions that are most effective for specific situations.

\section{PROPOSED GOVERNANCE MODEL}

In this section we introduce a business oriented governance model. This model adapts ideas of policy-driven management and optimization to build an autonomic cloud governance model. Although the approach is general enough for both SP and IP use, we here for clarity restrict the presentation of the governance model to an IP perspective.

Figure 1 shows the proposed business oriented governance model. The three main elements in the model are:

1) $B L O s$

The high level BLOs are the system inputs and they are expressed by high level objective functions and a set of constraints. An Objective function is technically a utility function and it is defined in terms of system parameters and it is subjected to constraints defined by system administrators. In a sample scenario, constraints can be thresholds for performance delivered or acceptable fractions (from an IP business point of view) of SLA violations.

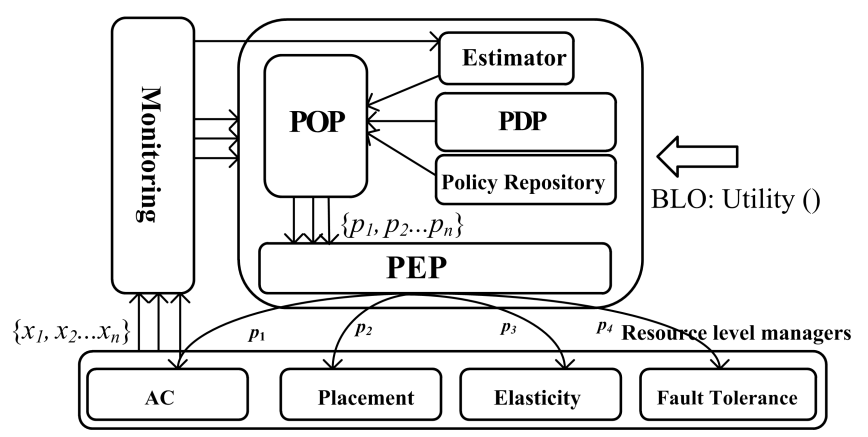

Fig. 1: Global view of business oriented governance model.

2) System parameters

Utility functions depend on a set of well-defined system parameters. System parameters can be values provided by the monitoring system, used for optimization towards the BLO or for assessing how well the constraints are met. These parameters can vary from number of deployed services, the avaialble non-allocated resources, number of violated SLA's and etc.

3) Resource level policies $\left\{p_{1}, \ldots, p_{n}\right\}$

Resource level policies are used to adjust the resource level managers' behaviors. The policies are imposed as constraints or objectives on resource level managers, derived from the optimization process in the governance model. These policies carry the governance level decisions to the resource level components. Resource level managers act not only based on their local objectives but also consider new constraints enforced as governance policies. In our model policies are adjustable values used in the resource level decision making process of each manager, and they aid to meet the high level objective. The policies are adjusted by the high-level manager after monitoring the full system behavior. The idea of using adjustable policies that change over time is motivated by the dynamic behavior of individual services and the whole system performance.

The core of the governance model consists of components described below:

1) The Policy Decision Point (PDP) is responsible for identifying which policy decisions need to be activated in order to achieve the high level objectives as well as how these policies should be applied.

2) The Policy Optimization Point (POP) is responsible for setting the policy values, and real time optimization and adaptation of policy values, based on dynamic changes in service demands and system behavior.

3) The Policy Enforcement Point (PEP) is responsible for selecting and enforcing the right policy from the policy set to the associated resource level managers.

4) The Policy repository stores policies that are used in the system.

5) The Estimator estimates the impact of changes in the 
system occurring by policy setting.

The governance process is a MAPE loop [29] (Monitor, Analyze, Plan, Execute) acting as an autonomic controller. Policy sets are defined by mapping each policy in the set to a resource manager. Each policy set is evaluated by the estimator that identifies the impact of possible changes. Resource level managers which include the policies into their decision making process, e.g., as constraints or thresholds, directly adjust their actions as policy values are modified. The first step is to evaluate the system state with respect to the defined objective function. The initial policy set adopted is selected based on previous data and system history. The optimization process is executed continuously in order to improve values for the controlling parameters in the policies as these values need to reflect changes in service and system behavior.

The result is adjusted behavior of the system through an implicit coordination between low level managers. This approach automatically shifts conflict resolution between the resource managers to the governance layer. Furthermore, conflict resolution is handled during policy setting avoiding conflicts during runtime. All effects of policy enforcement: changes in system parameters, system performance, and information about managed resources, are collected by the monitoring system for further analysis. Bellow, the model is illustrated in a sample scenario. For clarity, the scenario is kept significantly simpler than a full scale real world use case.

In this scenario, an IP's BLO is to maximize its revenue without losing reputation with its customers (which is formulated as keeping the SLA violation percentage less than 5\%). The utility function is given by equations 1 and 2 .

$\max \quad$ Profit $=\max ($ Revenue - Expenditure $)=\max U()$

where

$$
\begin{gathered}
U()=\sum_{i=1}^{n} R_{i}(t)-\bar{e}(t)-\sum_{i=1}^{n}\left(\hat{e}_{i}(t)+\check{e}_{i}(t)\right) \quad(e q .2) \\
\sum_{i=1}^{n} C_{i}(t) \leq p_{1} \times C_{\text {total }}(t) \quad \forall t \in T \quad(c .1) \\
v \leq 5 \% \quad(c .2)
\end{gathered}
$$

where $i=\{1 \ldots n\}$ is a service. The $R_{i}(t)$ is the revenue from service $i$ in time $t$. The $\bar{e}(t)$ is the fixed cost (e.g., including investment costs); The $\hat{e}_{i}(t)$ and $\check{e}_{i}(t)$ are running cost and the SLA violation penalty cost in time $t$ for service $i$. The $C_{i}(t)$ is the capacity required by the service $i$ during timeslot $t$. Finally, $C_{\text {total }}(t)$ is the total capacity available in the timeslot $t$, and $T$ is the set of time slots [18]. The value $v$ is the percentage of SLA violations, specified in the constraints to be less than $5 \%$.

As seen in (eq.1), maximizing profit is positively correlated with the number of deployed services and negatively associated with SLA violation penalties. Hence, the applied policies should be in line with deciding whether increasing the number of accepted services or avoiding costly penalties.
This relationship is independent of the fact that there in (c.2) is a constraint on the fraction of SLA violations. The main relationships between policies and managers are summarized in Table II.

TABLE II: Relationships between sample policies and low level managers

\begin{tabular}{|l|l|l|l|}
\hline Policy & Behavior & Associated with & Policy Value \\
\hline$p_{1}$ & $\begin{array}{l}\text { Provisioning } \\
\text { rate }\end{array}$ & Admission Control & $\begin{array}{l}25 \% \text { more than local } \\
\text { capacity (1.25) }\end{array}$ \\
\hline$p_{2}$ & $\begin{array}{l}\text { Consolidation } \\
\text { level per host }\end{array}$ & Placement & 4 VM/host \\
\hline
\end{tabular}

In the sample scenario, depicted in Table II, the behavior of admission controller is adjusted by tuning the policy $p_{1}$ for "provisioning rate". The policy $p_{1}$ is quantified by a value (25\%) representing that the $\mathrm{AC}$ is allowed to accept services whose aggregated maximum allowed capacity exceeds the local capacity by $25 \%$. The policy $p_{2}$ defines the maximum VM consolidation level per host. It defines the maximum number of VMs allowed to be deployed on a host in order to satisfy the BLO, in this example maximizing the revenue. The consolidation level also affects the elasticity controller (or vice versa), while changes in service demands should be supported by considering reasonable extra space on each host (or additional hosts available).SLA violations according to elasticity, result in paying costly penalties, which is in conflict with our BLO. Without loss of generality, we here assume only one type of VMs whereas a real use case would include a number of VM types with varying memory and CPU characteristics.

By enforcing policies, the AC and Placement engine are aligned. The result is that the entire system obtains the

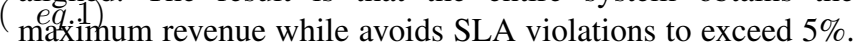
In a full-scale scenario, policies should also be defined for other resource level managers, like elasticity controller, fault tolerance engine, or data manager.MOreover some constant values in this example, like $\check{e}_{i}(t)$ can be a function in a full scale use case.

\section{CONCLUDING REMARKS}

This paper motivates the use of business oriented cloud governance to unify cloud resource management, striving towards an overall business level objective. The available solutions are reviewed with the main focus on perspectives on problem definition and formulation while introducing features and their significance. We also introduce a set of challenges for developing a business oriented governance model. A preliminary sketch of our proposed governance model is presented. Based on identified challenges, our future research will mostly focus on improving the model and making more comprehensive studies of BLO interpretation and policy mapping on low level managers.

We highlight that several challenges still need to be addressed in further studies, including:

1) Supporting a wide range of BLOs requires a broad understanding about various potential solutions to achieve 
each BLO. From a technical perspective, all the relationships between each possible actions of low level managers, the system parameters, and possible goals need to be defined. To define all these relationships is costly and difficult. Hence, adopting a technique that can automatically derive management policies and their relationships is of interest. Reinforcement learning and numerical or combinatorial optimization techniques are deemed suitable options, but a further analysis of these techniques in view of the governance model is still needed.

2) Exploring possible qualitative factors like trust, risk, and eco efficiency, to support more complex managerial objectives in the governance model is also under consideration. However, such factors increase the complexity of the governance process, since quantifying qualitative features like trust and risk is difficult. Thus, we first need to perform a cost/benefit analysis to select the appropriate quality factors to consider.

3) Extending and generalizing the governance model to be applied on other stakeholders like Service Providers(SPs) and brokers is also our future intention.

\section{ACKNOWLEDGMENT}

Financial support for this work is provided in part by the European Commission's Seventh Framework Programme ([FP7/2001-2013]) under grant agreement number 257115, OPTIMIS.

\section{REFERENCES}

[1] E. Elmroth and P. Gardfjäll, "Design and evaluation of a decentralized system for Grid-wide fairshare scheduling," in First International Conference on e-Science and Grid Computing, 2005., pp. 221-229, IEEE, 2006.

[2] A. Ferrer, F. Hernández, J. Tordsson, E. Elmroth, C. Zsigri, R. Sirvent, J. Guitart, R. Badia, K. Djemame, W. Ziegler, T. Dimitrakos, S. Nair, G. Kousiouris, K. Konstanteli, T. Varvarigou, B. Hudzia, A. Kipp, S. Wesner, M. Corrales, N. Forgó, T. Sharif, and C. Sheridan, "OPTIMIS: a Holistic Approach to cloud service Provisioning," 2010.

[3] M. Litoiu and M. Litoiu, "Optimizing resources in cloud, a SOA governance view," in Proceedings of the 2010 Workshop on Governance of Technology, Information and Policies, pp. 71-75, ACM, 2010.

[4] M. Litoiu, M. Woodside, J. Wong, J. Ng, and G. Iszlai, "A business driven cloud optimization architecture," in Proceedings of the 2010 ACM Symposium on Applied Computing, pp. 380-385, ACM, 2010.

[5] "Foundations of self-governing ICT infrastructures, FoSII." http://www.infosys.tuwien.ac.at/linksites/FOSII /index.htm.

[6] T. Pueschel, A. Anandasivam, S. Buschek, and D. Neumann, "Making Money With Clouds: Revenue Optimization Through Automated Policy Decisions," in 17th European Conference on Information Systems (ECIS 2009), Verona, Italy, pp. 355-367, 2009.

[7] J. Perez, C. Germain-Renaud, B. Kégl, and C. Loomis, "Utility-based reinforcement learning for reactive grids," in Autonomic Computing, 2008. ICAC'08. International Conference on, pp. 205-206, IEEE, 2008.

[8] J. Perez, C. Germain Renaud, B. Kégl, and C. Loomis, "Multi-objective reinforcement learning for responsive grids," Journal of Grid Computing, vol. 8, no. 3, pp. 473-492, 2010.

[9] H. Moon, Y. Chi, and H. Hacigumus, "SLA-Aware Profit Optimization in Cloud Services via Resource Scheduling," in 2010 6th World Congress on Services, pp. 152-153, IEEE, 2010.

[10] M. Goldszmidt, M. Budiu, Y. Zhang, and M. Pechuk, "Toward automatic policy refinement in repair services for large distributed systems," ACM SIGOPS Operating Systems Review, vol. 44, no. 2, pp. 47-51, 2010.
[11] D. Neumann, J. Stöesser, A. Anandasivam, and N. Borissov, "SORMA - Building an Open Grid Market for Grid Resource Allocation,” in 4th International Workshop, GECON 2007, Rennes, France, August 28, 2007, Proceedings, GECON, vol. 4685 of Lecture Notes in Computer Science, pp. 194-200, 2007.

[12] T. Püschel, N. Borissov, M. Macas, D. Neumann, J. Guitart, and J. Torres, "Economically Enhanced Resource Management for Internet Service Utilities," in Lecture Notes in Computer Science, (2007): The 8th International Conference on Web Information Systems Engineering (20Acceptance rate), pp. 335-348, 2007.

[13] J. Altmann, C. Courcoubetis, G. Stamoulis, M. Dramitinos, T. Rayna, M. Risch, and C. Bannink, "GridEcon: A market place for computing resources," Grid Economics and Business Models, pp. 185-196, 2008.

[14] J. Altmann, C. Courcoubetis, J. Darlington, and J. Cohen, "Gridecon - the economic-enhanced next-generation internet," in 4th International Workshop, GECON 2007, Rennes, France, August 28, 2007, Proceedings, GECON, vol. 4685 of Lecture Notes in Computer Science, pp. 188193, 2007.

[15] "Gridecon." http: / /www.gridecon.eu.

[16] M. Salehi and R. Buyya, "Adapting Market-Oriented Scheduling Policies for Cloud Computing," in Algorithms and Architectures for Parallel Processing (C.-H. Hsu, L. Yang, J. Park, and S.-S. Yeo, eds.), vol. 6081 of Lecture Notes in Computer Science, pp. 351-362, Springer Berlin / Heidelberg, 2010.

[17] J. Silva, L. Veiga, and P. Ferreira, "Heuristic for resources allocation on utility computing infrastructures," in Proceedings of the 6th international workshop on Middleware for grid computing, pp. 1-6, ACM, 2008.

[18] N. W. Paton, M. A. T. Aragão, K. Lee, A. A. A. Fernandes, and R. Sakellariou, "Optimizing utility in cloud computing through autonomic workload execution," IEEE Data Eng. Bull., vol. 32, no. 1, pp. 5158, 2009.

[19] D. M. Chess, A. Segal, and I. Whalley, "Unity: Experiences with a Prototype Autonomic Computing System," in ICAC '04: Proceedings of the First International Conference on Autonomic Computing (ICAC'04), (Washington, DC, USA), pp. 140-147, IEEE Computer Society, 2004.

[20] E. Manoel, S. Brumfield, K. Converse, M. DuMont, L. Hand, G. Lilly, M. Moeller, A. Nemati, and A. Waisanen, "Provisioning On Demand: Introducing IBM Tivoli Intelligent Think Dynamic Orchestrator ," 2003.

[21] R. Das, I. Whalley, and J. Kephart, "Utility-based collaboration among autonomous agents for resource allocation in data centers," in Proceedings of the fifth international joint conference on Autonomous agents and multiagent systems, pp. 1572-1579, ACM, 2006.

[22] J. Li, J. Chinneck, M. Woodside, M. Litoiu, and G. Iszlai, "Performance model driven QoS guarantees and optimization in clouds," in Proceedings of the 2009 ICSE Workshop on Software Engineering Challenges of Cloud Computing, pp. 15-22, IEEE Computer Society, 2009.

[23] J. Li, J. Chinneck, M. Woodside, and M. Litoiu, "Fast scalable optimization to configure service systems having cost and quality of service constraints," in Proceedings of the 6th international conference on Autonomic computing, pp. 159-168, ACM, 2009.

[24] V. Emeakaroha, I. Brandic, M. Maurer, and S. Dustdar, "Low Level Metrics to High Level SLAs-LoM2HiS framework: Bridging the gap between monitored metrics and SLA parameters in Cloud environments," in High Performance Computing and Simulation (HPCS), 2010 International Conference on, pp. 48-54, IEEE, 2010.

[25] M. Maurer, I. Brandic, V. Emeakaroha, and S. Dustdar, "Towards knowledge management in self-adaptable clouds," in 2010 6th World Congress on Services, pp. 527-534, IEEE, 2010.

[26] G. Tesauro, N. Jong, R. Das, and M. Bennani, "On the use of hybrid reinforcement learning for autonomic resource allocation," Cluster Computing, vol. 10, no. 3, pp. 287-299, 2007.

[27] C. Li and L. Li, "A distributed decomposition policy for computational grid resource allocation optimization based on utility functions," Microprocessors and Microsystems, vol. 29, no. 6, pp. 261-272, 2005.

[28] R. Bahati and M. Bauer, "Adapting to run-time changes in policies driving autonomic management," in Fourth International Conference on Autonomic and Autonomous Systems, pp. 88-93, IEEE, 2008.

[29] IBM, "An architectural blueprint for autonomic computing," white paper, 2004. 